\title{
Cardiac arrhythmia in Wilson's disease: An oversighted and overlooked entity!
}

\author{
Bhupender Kumar Bajaj, Ankur Wadhwa, Richa Singh, Saurabh Gupta
}

Department of Neurology, PGIMER and Dr. RML Hospital, New Delhi, India

\begin{abstract}
Wilson's disease is a multisystem disorder which manifests with hepatic, neurological, musculoskeletal, hematological, renal, and cardiac symptoms. The hepatic and neurological manifestations often overshadow the other system involvement including cardiac symptoms and signs, which may prove fatal. We report a case of a young female who presented with progressive parkinsonian features and dystonia for around 4 months followed 2 months later by the complaint of episodes of light-headedness. She was diagnosed to have Wilson's disease based on the presence of Kayser-Fleischer ring and laboratory parameters of copper metabolism. Electrocardiography of the patient incidentally revealed $2^{\text {nd }}$ degree Mobitz type-1 atrioventricular block explaining her episodes of light-headedness. She was started on penicillamine and trihexyphenidyl. The heart block improved spontaneously. Cardiac autonomic function tests including blood pressure response to standing and heart rate response to standing were observed to be normal. We review the literature on cardiac manifestations of Wilson's disease and emphasize that patients with Wilson's disease should be assessed for cardiac arrhythmia and cardiac dysfunction as these may have therapeutic and prognostic implications.
\end{abstract}

Key words: Autonomic function, cardiac arrhythmia, heart block, hepatolenticular degeneration, Wilson's disease

\section{Introduction}

Wilson's disease is a multisystem disorder which manifests with hepatic, neurological, musculoskeletal, hematological, renal, and cardiac symptoms. When treated early, the chelation therapy may lead to reversal of symptoms. Death in Wilson's disease is generally from hepatic failure, bleeding esophageal varices, or intercurrent infection in those with severe neurological involvement leading to bedridden status. The hepatic and neurological manifestations often overshadow the other system involvement including cardiac symptoms and signs. We report a case of a young female who had heart block which was detected incidentally when the electrocardiogram (ECG) was done. This

Address for correspondence:

Dr. Bhupender Kumar Bajaj, Department of Neurology, PGIMER and Dr. RML Hospital, New Delhi - 110 001, India.

E-mail: docbajaj@yahoo.co.in

\begin{tabular}{|l|l|}
\hline \multicolumn{2}{|c|}{ Access this article online } \\
\hline Quick Response Code: & Website: \\
\hline & www.ruralneuropractice.com \\
\cline { 2 - 2 } & \\
\hline
\end{tabular}

led to the explanation for her persistent complaint of intermittent vague sense of light-headedness, weakness, and blurring of vision amidst the florid neurological manifestations.

\section{Case Report}

A 14-year-old female born of consanguineous marriage presented with progressive bradykinesia, generalized dystonia, and dysarthria of around 4 months. She was observed to have vacuous smile all the time by her mother and had reduced interest in day-to-day activities. She had become irritable and tearful. She complained of intermittent vague sense of weakness accompanied by blurring of vision and falls for around 2 months. The patient had a past history of two episodes of jaundice. On examination, her pulse was 78/min, regular and rhythmic, and blood pressure was $100 / 60 \mathrm{mmHg}$ with no postural

This is an open access article distributed under the terms of the Creative Commons Attribution-NonCommercial-ShareAlike 3.0 License, which allows others to remix, tweak, and build upon the work non-commercially, as long as the author is credited and the new creations are licensed under the identical terms.

\section{For reprints contact: reprints@medknow.com}

How to cite this article: Bajaj BK, Wadhwa A, Singh R, Gupta S. Cardiac arrhythmia in Wilson's disease: An oversighted and overlooked entity!. J Neurosci Rural Pract 2016;7:587-9. 
fall. Ocular examination revealed Kayser-Fleischer ring which was confirmed by slit lamp examination. She had hypomimia with grinning face. Her speech was hypophonic and dysarthric with ability to utter only a few comprehensible words. Saliva continuously drooled from her mouth. She could walk with assistance only. The patient did not have any ocular movement abnormalities or cerebellar and pyramidal signs. Sensory examination was normal. Laboratory examination showed hemoglobin of $10.4 \mathrm{~g} / \mathrm{dl}$, total leukocyte count of $5800 / \mathrm{mm}^{3}$, and platelet count of $80,000 / \mathrm{mm}^{3}$. Liver function and kidney function tests were observed to be within normal limits. The patient had low serum ceruloplasmin $(12 \mathrm{mg} / \mathrm{dl}$; normal: $20-40 \mathrm{mg} / \mathrm{dl})$ and increased $24 \mathrm{~h}$ urinary copper (45 $\mu \mathrm{g}$; normal 20-40 $\mu \mathrm{g}$ ). Electrocardiography of the patient done on subsequent day during admission revealed $2^{\text {nd }}$ degree Mobitz type- 1 atrioventricular nodal block [Figure 1]. Echocardiography reported to be normal and ultrasonography of the abdomen showed mild splenomegaly with normal liver echotexture and portal vein diameter. Brain computed tomographic scan revealed bilateral hypodensities in putamen, caudate, thalami, periaqueductal mid brain, and tegmental pons [Figure 2]. Brain magnetic resonance imaging showed $\mathrm{T} 1$ hypointense and $\mathrm{T} 2$ hyperintense lesions in bilateral putamen, caudate, thalami, periaqueductal midbrain, and tegmental pons [Figure 3]. She was started on penicillamine and trihexyphenidyl. Holter monitoring started a few days later did not show a significant cardiac arrhythmia requiring intervention. Her symptoms of intermittent sense of light-headedness, weakness, and blurring of vision improved over the week. Cardiac autonomic function tests including blood pressure response to standing and heart rate response to standing were observed to be within normal limits. Heart rate response to Valsalva maneuver could not be tested due to inability of the patient to perform Valsalva maneuver.

\section{Discussion}

The evaluation and management of cardiac involvement of patients with Wilson's disease is not adequately reported in literature. The term "hepatolenticular degeneration" for Wilson's disease belies the systemic involvement of various organ systems of the body by the copper toxicity. While the hepatic and the neurological manifestations are the prominent clinical features of the disease, other organ involvement including that of heart may have prognostic and therapeutic implications. Kuan evaluated 53 consecutive patients with Wilson's disease and described four modes of cardiac manifestation including cardiac arrhythmia, cardiomyopathy, cardiac death, and autonomic dysfunction. ${ }^{[1]} \mathrm{He}$ observed electrocardiographic abnormalities in 34\% and asymptomatic orthostatic hypotension in 19\% of his patients. Abnormal response to Valsalva maneuver was detected in $33 \%$ of the patients. The author reported two cardiac deaths, of which one patient died of ventricular fibrillation and the other died of cardiomyopathy. Ever since this report, there have been only scant studies on the prevalence and prognostic implications of cardiac Wilson's disease. Hlubocká et al. evaluated 42 patients of Wilson's disease and 42 age- and sex-matched healthy subjects with echocardiography and 24 h ECG Holter monitoring. ${ }^{[2]}$ They observed an increased thickness of interventricular septum and left ventricular posterior wall in patients with Wilson's disease. The authors detected electrocardiographic abnormalities in 10 of the 23 patients who underwent Holter monitoring. They observed that the supraventricular tachycardia and ectopics were the most frequent abnormalities on Holter monitoring. The largest two series of Wilson's disease till date reported non-neurological presentation in $22.3 \%$ and $55.7 \%$ of Wilson's disease patients. ${ }^{[3,4]}$ Interestingly, neither of these mentions of comprehensive assessment of possible cardiac manifestations. In a series of fifty patients evaluated by electrocardiography, 15 were reported to have at least one abnormality on the ECG. ${ }^{[5]}$ The authors observed sinus bradycardia, tachycardia, bifid P wave, ST elevation or depression, $\mathrm{T}$ inversion, ventricular premature beat, and prominent $\mathrm{u}$ wave as the electrocardiographic abnormalities. There is a report of tricuspid regurgitation in a patient with hepato-neurological presentation of Wilson's disease. ${ }^{[6]}$ The report does not conclusively establish that the tricuspid regurgitation is in any way due to copper toxicity. Our patient had complaints of episodic light-headedness which were possibly due to the heart block. Echocardiography of the patient did not reveal

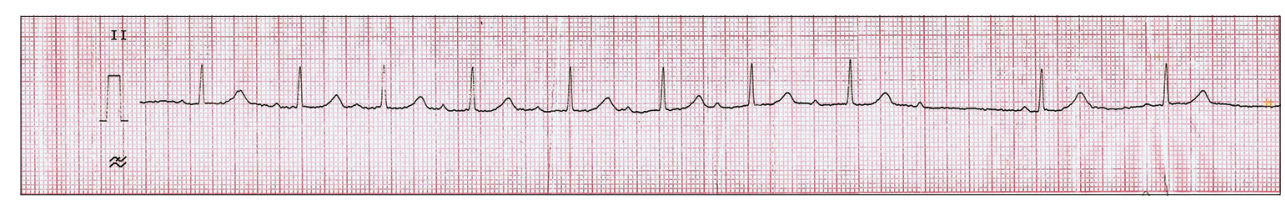

Figure 1: Long lead II of electrocardiograph showing $2^{\text {nd }}$ degree heart block Mobitz type-1 


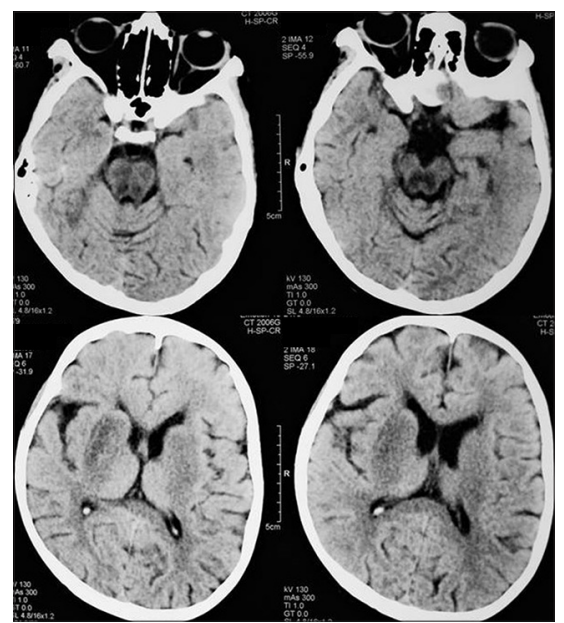

Figure 2: Computed tomographic brain showing hypodensities in the putamen, caudate, thalami, midbrain, and tegmental pons

any abnormality. Holter monitoring done a few days later failed to show significant cardiac arrhythmia. The $2^{\text {nd }}$ degree heart block in our patient recovered without any intervention except the initiation of trihexyphenidyl and penicillamine for symptomatic treatment of dystonia and chelating therapy for Wilson's disease. It could have been a spontaneous improvement. Recently, myocardial integrated ultrasonic backscatter has been reported to detect early changes in myocardium of patients with Wilson's disease. ${ }^{[7]}$

\section{Conclusion}

Cardiac evaluation of patients with Wilson's disease should be a part of routine evaluation of the patients as cardiac arrhythmia and cardiac dysfunction may have therapeutic and prognostic implications.

\section{Financial support and sponsorship}

Nil.

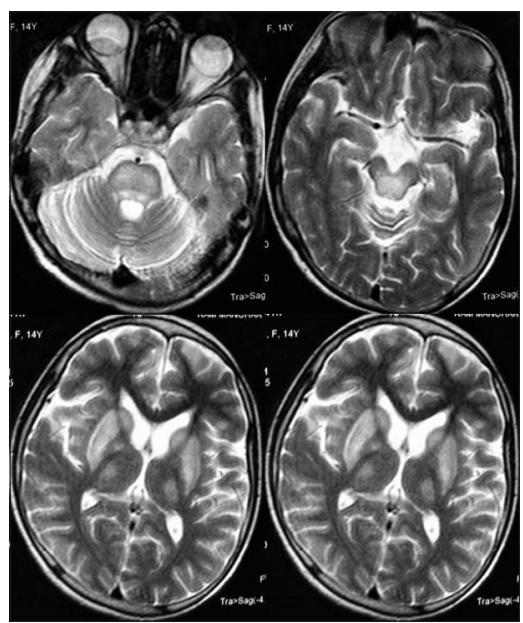

Figure 3: Magnetic resonance imaging brain showing T2 hyperintense lesions in bilateral putamen, caudate, thalami, midbrain, and tegmental pons

\section{Conflicts of interest}

There are no conflicts of interest.

\section{References}

1. Kuan P. Cardiac Wilson's disease. Chest 1987;91:579-83.

2. Hlubocká Z, Marecek Z, Linhart A, Kejková E, Pospísilová L, Martásek P, et al. Cardiac involvement in Wilson disease. J Inherit Metab Dis 2002;25:269-77.

3. Walshe JM. Wilson's disease. In: Vinken PJ, Bruyn GW, Klawans HL, editors. Handbook of Clinical Neurology. Vol. 49. Amsterdam: Elsevier; 1986. p. 223-38.

4. Taly AB, Meenakshi-Sundaram S, Sinha S, Swamy HS, Arunodaya GR. Wilson disease: Description of 282 patients evaluated over 3 decades. Medicine (Baltimore) 2007;86:112-21.

5. Meenakshi-Sundaram S, Sinha S, Rao M, Prashanth LK, Arunodaya GR, Rao S, et al. Cardiac involvement in Wilson's disease - An electrocardiographic observation. J Assoc Physicians India 2004;52:294-6.

6. Fatima J, Karoli R, Siddiqui Z, Chandra A, Jain V. Hepatolenticular degeneration - Is the term too narrow to explain Wilson's disease? J Assoc Physicians India 2014;62:531-3.

7. Arat N, Kacar S, Golbasi Z, Akdogan M, Kuran S. Myocardial integrated ultrasonic backscatter for early detection of cardiac involvement in patients with Wilson disease. Turk J Gastroenterol 2014;25:678-84. 